\title{
Erratum
}

\section{Analysis and Design of Grating Couplers}

T. Tamir and S. T. Peng

Department of Electrical Engineering

Polytechnic Institute of New York, Brooklyn, N.Y. 11201, USA

Appl. Phys. 14, 235-254 (1977)

In Table $2, j_{n}$ and $E_{z p}$ should read as follows

$$
\begin{array}{r}
j_{n}=-i \omega \varepsilon_{0} \varepsilon_{n}(z) V_{g}(z), \quad \text { (for both } T E \text { and } T M \text { modes) } \\
E_{z p}=-\sum_{n} \frac{\beta_{n}}{\omega \varepsilon_{0} \varepsilon_{u}} I_{n} \exp \left(i \beta_{n} x\right)-\sum_{n} \frac{v_{n}(z)}{i \beta_{n}} \exp \left(i \beta_{n} x\right) \\
\text { (for } T M \text { modes only) }
\end{array}
$$

